\title{
Research on the Technology of On-line Audit Warning in Big Data Environment
}

\author{
Sui Jie ${ }^{1, a}$, Zuochun Fang ${ }^{2, b}$ \\ ${ }^{1}$ Department of Economics and Management, Tianjin Normal University of Vocation and \\ Technology, Tianjin, 300222, China \\ ${ }^{2}$ Department of Business Administration, Tianshi College, Tianjin, 301700, China \\ aemail: suijie0625@126.com, bemail: 63404438@qq.com
}

\section{Keywords: Audit Warning; On-line Auditing; Big Data}

\begin{abstract}
Under the background of big data, information technology is not only the object of audit work, but also the important tool of it. According to the principle and influence of on-line audit and characteristics of audit early warning technology, firstly, we introduce the necessity of on-line audit in big data environment, and then from the rule base and integrity detection of data, two aspects, we analyze the key technology of audit early warning mode. Finally, the suggestion is put forward how to promote the technology of the on-line audit early warning.
\end{abstract}

\section{Introduction}

With the big data era coming, audit has changed a lot. The mode of on-line audit appears as a bridge between audit institutions and the audited company, which concerns information collecting and processing, analysis and evaluation, audit conclusion and so on.

At present, on-line audit implemented in China is not embedded audit but rather split continuity audit [1]. The essential difference between the two methods is whether the audit module is embedded in the information system of the audited company. The split continuity audit is a mode of amassing information with the help of network in order to judge the business authenticity of the audited organization and provide the doubtful data and information for on-line audit. In the technology aspect, it mainly includes data acquisition, data conversion, data transmission and data storage and so on. As an important part of the on-line audit, data collection largely determines audit quality. As a result, it is essential for the audited company to adopt some precautions during collecting data, which can guard against risks and improve the efficiency of audit work. Especially, lots of on-line audit projects are actively carried out, such as financial institutions on-line audit projects, customs audit projects and government financial on-line audit projects, etc.[2]. Therefore, it is important theoretical and practical significance of research on the technology of on-line audit warning in big data environment.

\section{Principle and Influence of On-line Audit in Big Data Environment}

On-line audit is able to realize remote inspection and supervision of the property, whether it's authentic and integral in the audited company. These functions must be played with the help of the network to collect the electronic data. The technology of on-line audit is implemented through the following steps: (1) through the server, auditors can complete data collection of the audited company; (2) the data is passed to the audit institution for storage; (3) auditors analyze and deal with the data, and then find audit trails.

The impact of big data on the internet audit is reflected in four main areas, which are audit scope, audit procedure, audit evidence and risk assessment. Firstly, under big data environment, the audited organization is a massive data collection. So the audit work cannot infer the limited data from the small sample, but the relevant data is analyzed comprehensively. On-line audit is able to expand work scope from the whole to the local, so that audit data analysis work becomes more comprehensive and credible. Secondly, under the background of big data, audit procedures should 
be improved as follows: (1) audit work involves mass, diverse, complete data; (2) auditors attempt to use artificial intelligence to collect data, text information for business truth or query risk; (3) auditors can expand the scope of the data, gather other sources of information data. Thirdly, under the background of big data, audio, documents, pictures, etc. will become audit evidence. SQL, traditional relational database, cannot meet the audit needs [3]. Finally, auditors can use many risk assessment methods, such as cluster analysis, which the data are grouped according to the characteristics for finding data association and reducing audit risk by data mining.

\section{Early Warning Model of On-line Audit in Big Data Environment}

When audit work into the audit implementation stage, there is an important part of the audit warning, which includes two parts, the pre-audit system and the pre-audit report system. The pre-audit system is automatically activated after data acquisition with the analysis of the prior placement and the early warning rule, and then it filters inadequate and questioned data. The pre-audit report system is automatically activated after the first phase, which reports the auditors doubtful data stored in the database, in order to facilitate the audit staff to conduct professional judgment and carry out the next step of the audit work. Specific processes are shown in Figure 1.

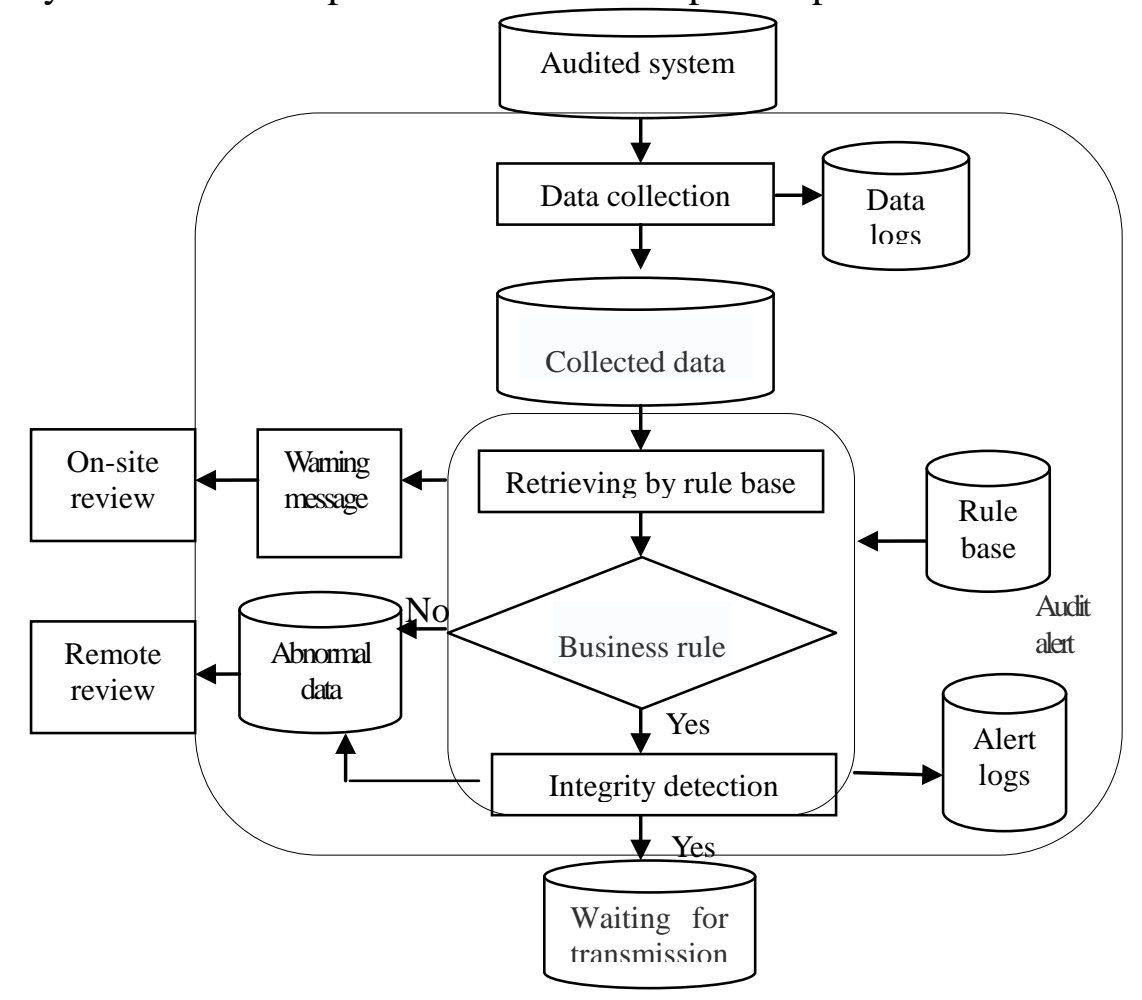

Fig.1. Early Warning Model of On-line Audit

From Figure 1, we find the model, which is not comply with pre- auditing the data by business rules, but also detects fragmentary data, so that auditors make decision of further work.

\section{Key Technology of the Early Warning of On-line Audit}

The key technology of the early warning of on-line audit includes rule base, detection of incomplete data, dealing with incomplete data etc.[6]. Below we focus on two important technologies, which are rule base and d checking the integrity of transmitted data.

Rule base is an important link in early warning model of on-line audit, which mainly includes data acquisition, data filtering, system analysis log and monitoring information conversion strategy, etc.[4][5]. The configuration principle is that the setting conditions (one or more) will trigger a specific operation, when the condition is satisfied. For instance, some causal production rules, the general use of the IF-P-THEN-Q, P represents precondition, and Q is the conclusion or behavior, if 
P can be fulfilled, Q represents the execution results or behavior, the formula is as follows:

$$
R_{k}: \stackrel{n}{O R}\left(\underset{i=1}{\operatorname{AND}} \underset{j=1}{E_{i j k}}\right) \rightarrow D_{k}
$$

In the type(1), m, $n>1, k=1,2 \cdots r, R_{k}$ stands for the $K$ rule, $D_{k}$ stands for the $K$ conclusion. In the application, the rule base order can be defined or modified according to the specific industry, business features, so that the audit early warning model can adapt to operation and become more adaptive and practical.

Detection the integrity of transmitted data, in Figure 1, the on-line audit system collects data through the rule base detection and after meeting the business rules, it will start to detect the data. In this module, if the detection algorithm in the database is fragmentary, the data is not cleaned. But if the record isn't fragmentary, it is passed to the detection module for further detecting and determined whether it can be by rule base. It is deleted, if not available, otherwise it is transmitted to the database after being completed records. The integrity of the detection record is estimated by the percentage of missing field values and the function is as follows:

$$
\begin{aligned}
& X=\left\{x_{1}, x_{2} \cdots x_{n}\right\} \\
& X M=\frac{\alpha}{n}\langle\lambda, \lambda \in[0,1]
\end{aligned}
$$

In the type(2), $X$ stands for a record, $x_{1} \ldots x_{n}$ are $\mathrm{n}$ fields of $X$; in the type(3), $\alpha$ stands for missing value of $X, X M$ is the ratio of $X$ values missing, $\lambda$ is the threshold of ratio of $X$ values missing.

According to the above analysis, pseudo code of incomplete data detection is described as:

(1) FOR $i=1$ TO t, t means the total number of data sheets

(2) FOR i $=1$ TO n

(1) IF $R_{i}\left(a_{j}\right)$ is NULL Or $R_{i}\left(a_{j}\right)=R_{i}\left(\left(a_{j}(\right.\right.$ default $\left.\left.)\right)\right)$, then this record is incomplete data

(2) END IF

(3) END

(4) END

Auditors can deal with the lack of data by the method of artificial treatment, average substitution, etc.. The former method is mainly aimed at the absence of important attributes of data or data is not large, and then the other is based on the average value of the property missing value.

\section{Conclusion and Recommendations}

Compared with the traditional audit mode, on-line audit work constantly and real-time with reducing audit risk. The early warning model of audit can improve audit frequency and realize the remote audit purpose. As audit test of embedded module, the audit alert model improves the value of audit report from identification and review of each business data through the rule base and the detection of integrity data.

Using the audit alert model and doing well the on-line audit work, it needs to be equipped with data analysis personnel and professional analysis team after a long training and running. In addition, on-line audit work involves many processes, which must be adjusted and optimized by the operating conditions and industry characteristics of the audited company. Therefore, the on-line audit needs to increase amount of investment from human, technology, and fund, so that the on-line audit technology can be improved and developed under China's big data circumstances. 


\section{Acknowledgement}

In this paper, the research was sponsored by the Foundation of Tianjin Normal University of Vocation and Technology (Project No. KYQD15003).

\section{References}

[1] Chensun, Mai Chaocheng, Yu Huichuan. The effect of export tax rebates on export performance; Theory and evidence China [J]. China Economic Review,2006,17(2):226-235.

[2] Stanciu V. On-line Audit[J]. International Journal of Academic Research in Accounting, Finance and Management Sciences, 2013, 3(1): 231-237.

[3] Clarke P, Ailshire J, Melendez R, et al. Using Google Earth to conduct a neighborhood audit: reliability of a virtual audit instrument[J]. Health \& place, 2010, 16(6): 1224-1229.

[4] Porter B, Simon J, Hatherly D. Principles of external auditing[M]. John Wiley \& Sons, 2014.

[5] Yang K, Jia X. Data storage auditing service in cloud computing: challenges, methods and opportunities [J]. World Wide Web, 2012, 15(4): 409-428.

[6] Ruan H M, Lei C L. Fine-Grained Audit Privilege Control for Integrity Audit on Cloud Storage[C] Information Security (ASIA JCIS), 2014 Ninth Asia Joint Conference on. IEEE, 2014: 156-163. 